\title{
Clinicopathological Analysis between IgG4-related and Non-IgG4- related DiseasesOccurring in Various Organs and Tissues: Re- evaluation of ComprehensiveDiagnostic Criteria and Usefulness of Interleukin-6 Immunostaining
}

\author{
Xipeng Wang ${ }^{1,2^{\star}}$, Yuyu Sun ${ }^{1}$, Wei Liu' ${ }^{1}$, Weiguo Zhang ${ }^{2}$ \\ ${ }^{1}$ Department of Orthopaedic Surgery, Affiliated Hospital 2 of Nantong University, Nantong 226001,China; \\ ${ }^{2}$ Department of Orthopaedic the center hospital of Wuhan, Wuhan 430010, China
}

*Corresponding Author: Xipeng Wang MD,PhD, Department of Orthopaedic Surgery,Affiliated Hospital 2 of Nantong University, Nantong 226001,China. E-mail: tigerwxp@ foxmail.com

Received February 28, 2019; Accepted March 17, 2019

Comprehensive diagnostic criteria for IgG4-related diseases are widely accepted. However, clinicopathological features of probable and possible Ig4-related and non-IgG4-related diseases, which are subdivided by comprehensive diagnostic criteria, are not fully understood.IgG4-related disease (IgG4-RD) is a recently identified systemic fibro-sclerotic disease of unknown etiology. It exhibits multiple organ involvement,causes symmetrical or asymmetrical organomegaly, and forms nodular or hypertrophic lesions concurrently or metachronously. IgG4-RD has several typical characteristics such as predominant occurrence in middle-aged men; presence of antinuclear antibodies; high incidence of allergic diseases; eosinophilia; elevation of serum gammaglobulins, IgG, IgG4, and IgE; and good response to steroid therapy. Histologically, IgG4-RD is characterized by lymphoplasmacytic infiltration,lymphoid follicle formation, eosinophil infiltration,IgG4-positive plasma cell infiltration, storiform fibrosis (SF), and obliterative phlebitis $(O P)(1,2)$. Useful diagnostic tool to distinguish between IgG4-related and non-IgG4-related diseases and prediction of therapeutic responsiveness for IgG4-related disease is required. The purpose of this study is to clarify these diagnostic problems.

Methods: The clinicopathological features and expression of interleukin- 6 and glycoprotein 130 were analyzed in 94 and 34 cases of IgG4-related and non-IgG4-related diseases, respectively.

Results:Probable IgG4-related cases showed decreased plasma cell number, increased eosinophils and mast cell numbers, and marked fibrosis. Marked fibrosis and inflammatory cell infiltration were not observed in possible IgG4-related cases. Although most non-IgG4-related cases matched the comprehensive diagnostic criteria, completely or partially, could be easily differentiated based on histological findings, multicentric Castleman's disease exhibited findings closely similar to those of IgG4-related disease. Immunohistochemical expression of interleukin-6 in IgG4-related disease was significantly lower than that in multicentric Castleman's disease. Comprehensive diagnostic criteria could be effectively applied to IgG4-related disease occurring in various organs and tissues and was applied to biopsied and surgically resected specimens, although histological information was limited in the biopsied specimen. Relapsed cases after steroid therapy showed a higher incidence of multiple organ involvement and lower incidences of storiform fibrosis and obliterative phlebitis than the non-relapsed cases.

Conclusion:Probable IgG4-related disease was considered as the advanced phase of definite IgG4-related disease. Possible IgG4-related disease had to undergo repeat biopsy or surgical excision of other organs. Careful consideration of clinical information and immunostaining for interleukin-6 may be crucial to distinguish between IgG4-related and multicentric Castleman's diseases. It is useful to assess the fibrosis grade to evaluate the disease progression and the therapeutic responsiveness for IgG4- related disease occurring in various organs and tissues.

Key Words: immunity,IgG4-related disease, comprehensive diagnostic criteria, fibrosis grade, IL-6 


\section{Introduction}

IgG4-related disease (IgG4-RD) is a recently identified systemic fibro-sclerotic disease of unknown etiology. It exhibits multiple organ involvement,causes symmetrical or asymmetrical organomegaly, and forms nodular or hypertrophic lesions concurrently or metachronously. ${ }^{1}$ IgG4-RD has several typical characteristics such as predominant occurrence in middle-aged men; presence of antinuclear antibodies; high incidence of allergic diseases; eosinophilia; elevation of serum gammaglobulins, IgG, IgG4, and IgE; and good response to steroid therapy. ${ }^{1}$ Histologically, IgG4-RD is characterized by lymphoplasmacytic infiltration, lymphoid follicle formation, eosinophil infiltration,IgG4-positive plasma cell infiltration, storiform fibrosis (SF), and obliterative phlebitis (OP). ${ }^{2}$

In 2012, Umehara et al. proposed the following comprehensive diagnostic criteria (CDC) for IgG4-RDof various organs and tissues: 1) clinical examination shows characteristic diffuse/ localized swellings or masses in single or multiple organs; 2) hematological examination shows elevated serum IgG4 (S-IgG4) concentrations ( $\geqslant 135 \mathrm{mg} / \mathrm{dL}$ ); and 3) histological examination shows a) marked lymphocyte and plasma cell infiltration and fibrosis and b) infiltration of IgG4-positive plasma cells, particularly with IgG4positive plasma cell/IgG-positive plasma cell ratios (IgG4/IgGratios) $>40 \%$ and $>10$ IgG4-positive plasma cells/ high power field (IgG4/HPF). Definite IgG4-RD is defined as the presence of 1,2 , and 3; probable IgG4-RD as the presence of 1 and 3; and possible IgG4-RD as the presence of 1 and 2. Differential diagnosis is necessary from other disorders, including sarcoidosis, Castlemans's disease, Wegener's granulomatosis, lymphoma, and cancer. ${ }^{3}$

CDC for IgG4-RD has been widely accepted.Although the histological diagnostic scheme of IgG4-RDwas proposed by a set of cut-off points that is specific to each organ, ${ }^{2}$ clinicopathological features of probable, possible, and non-IgG4-RDs are not clear.Therefore, Okazaki et $\mathrm{al}^{4}$ reported that organ-specific criteria $^{5,6}$ should be used concurrently in the probable or possible cases. In addition,clinicopathological analysis of IgG4-RD occurring in various organs and tissues and clinicopathological analysis of single or multiple lesions in IgG4-RDhave not been sufficiently examined. There are increasing opportunities to diagnose IgG4-RD based on biopsied specimens, since a minimally invasive procedure is required. Moriyama et $\mathrm{al}^{7}$ reported the diagnostic utility of labial salivary gland biopsy in IgG4-RD. However,diagnostic usefulness of biopsied specimens is not examined in other various organs and tissues.

The steroid is a first-line therapy in IgG4-RD, and it is performed following pathological diagnosis; Novel therapeutic approaches are proposed in the field ofIgG4-RD. ${ }^{8}$ It is necessary to

This is an open access journal, and articles are distributed under the terms of the Creative Commons Attribution-NonCommercial-ShareAlike 4.0 License, which allows others to remix, tweak, and build upon the work non-commercially, as long as appropriate credit is given and the new creations are licensed under the identical terms.

\section{For reprints contact: weda-h@weda-h.org}

How to cite this article: Wang XP, Sun YY, Cui ZM, Liu W. Clinicopathological Analysis between IgG4-related and Non-IgG4related DiseasesOccurring in Various Organs and Tissues: $\operatorname{Re}\rceil$ evaluation of ComprehensiveDiagnostic Criteria and Usefulness of Interleukin-6 Immunostaining. J ADV HEALTH 2019; 1(2): 122-131. find the factors which influence on the therapeutic responsiveness to theIgG4-RD. Some cases of non-IgG4-RD such as autoimmune diseases, multicentric Castleman's disease (MCD), or malignant tumors exhibit overproduction of interleukin -6 (IL-6), leading to IgG4-positive plasm cell infiltration and serum IgG4 elevation. ${ }^{9}$ In particular, MCD is challenging to distinguish from IgG4-RD because of histological similarity.10 Therefore, immunohistochemical analysis for IL-6 and glycoprotein 130 (gp130), which transmits an IL-6signal into the cell together with the IL-6 receptor, ${ }^{11}$ was performed in order to evaluate whether it is possible to distinguish between IgG4-RD and MCDon the tissue sections.

\section{Materials and methods}

\section{Specimens analyzed}

A total of 94 IgG4-RD cases and 34 non-IgG4-RD cases were retrospectively evaluated. Biopsied and surgically resected specimens of IgG4-RD and nonIgG4-RD were collected from the cases of Second Affiliated Hospital of Nantong University ( $\mathrm{n}=$ 30) and from cases consulted from 2008 to $2018 \quad$ ( $n=98$ ). This study was approved by the institutional ethics committee of Nantong University. The male:female ratio was 62:32 among IgG4-RD cases and 24:10 among non-IgG4-RD cases, and patients in the former group were older than those in the latter group $(63.0 \pm$ 12.1 years vs $53.0 \pm 18.8$ years; $p=0.0072$ ). The age of all the patients are above 30 years were in either group.

Although non-IgG4-RD groups are clinically suspected as IgG4-RD because of organ enlargement tor serum IgG4 elevation, it is possible to distinguish between the two based on their clinical and histopathological findings. In this study, non-IgG4-RD is defined as the disease that fulfills condition 1)and satisfies conditions 2) or 3) or both of the CDC for IgG4-RD.

There were a total of 177 lesions in the IgG4-RDgroup and 37 lesions in the non-IgG4-RD group.Ninety-four cases diagnosed with the biopsied specimen and 34 cases diagnosed with surgically resected specimen were evaluated. The non-IgG4-RD cases included 14 Castleman's disease (6 multicentric, 8unicentric), 7 malignant lymphoma (6 MALToma, langioimmunoblastic T-cell lymphoma), 3 non- specific reactive lymphadenopathy, 2 chronic sialoadenitis, 2Warthin's tumor, 1 Crow-Fukase disease, 1 Kimura'sdisease, 1 interstitial nephritis, 1 membranous nephropathy, 1 retroperitoneal fibrosis, and linflammatory myofibroblastic tumor.

All tissue samples were fixed in $10 \%$ neutral-buffered formalin and embedded in paraffin. Sections were stained with hematoxylin and eosin (HE),toluidine blue ( $\mathrm{pH}$ 7.0) to count numbers of mast cells, and elastica van Gieson to detect OP.

\section{Immunohistochemistry}

Immunohistochemical reactions were performed on 4-5 $\mu$ m-thick formalin-fixed and paraffin-embedded sections. All stains were performed automatically(BenchMark GX, Ventana Medical System, Tucson,Arizona, USA) using the standard immunoperoxidase method, and 3,3'-diaminobenzidine was used as a chromogen. Slides were immunostained using either an anti-human IgG4 monoclonal antibody (clone HP6025,1:400 dilutions; Binding Site, Birmingham, UK), an anti-human IgG polyclonal antibody (1:5,000 dilution;DAKO, Carpinteria, CA, USA), an anti-human IL-6monoclonal antibody (clone 10C12, 1:50 dilution;Leica Microsystems, Wetzlar, Germany), or an anti-human gp130 
polyclonal antibody (1:500 dilution; Santa Cruz Biotechnology, San Diego, CA, USA) with incubation for $30 \mathrm{~min}$ at $37^{\circ} \mathrm{C}$. Antigen retrieval with proteinase $\mathrm{K}(85 \mu \mathrm{g} / \mathrm{ml}$, DAKO) for $6 \mathrm{~min}$ was performed for the anti-human IgG polyclonal antibody.

\section{Analyzed factors and assessment of light microscopic findings}

Clinicopathological features in IgG4-RD and nonIgG4-RD were analyzed with the following factors: 1)age and sex, 2) sites involved, 3) S-IgG4 level, 4)histological features, 5) IgG4/IgG ratios and IgG4/HPF, 6) lymphocyte-infiltration grade and infiltration of eosinophils and mast cells, 7) fibrosis grade, and 8)presence of SF and OP. Clinicopathological features in definite IgG4-RD were analyzed with the following factors: 9) biopsied and surgically resected specimens,10) single and multiple organ involvement, and 11)relapse after steroid therapy. In addition, 12)clinicopathological and immunohistochemical comparison in definite IgG4-RD and MCD were also analyzed. All histological features were measured under400x magnification (objective, Plan Apo 40x/0.95, ocular; CFI 10x/22; Nikon ECLIPSE MicroscopeE600, Nikon Instech Co., Ltd., Tokyo). To evaluateIgG4/IgG ratios, IgG4/HPF, infiltration of eosinophils and mast cells, 5 HPFs were selected from prominent inflammatory areas and number of cells were counted under microscopy. The average number of IgG4-positive plasma cells and IgG-positive plasma cells/5HPF and the total number of eosinophils and mast cells /5 HPFs were calculated. Plasma cells that could be clearly identified by the presence of a nucleus and cytoplasm were counted. The status of fibrosis was graded according to four categories ranging from 0 to 3 , where $0=$ no fibrosis, 1 = slight fibrosis, 2 = septal fibrosis, and 3 = dense fibrosis or sclerosis (Fig.1a-d). The lymphocyte infiltration was defined using the following grading scheme: $0=$ very slight infiltration of lymphocytes, 1 = mild infiltration of lymphocytes, 2 = lymphocyte aggregation without germinal centers, and $3=$ lymphoid follicles with germinal centers (Fig. 2a-d). IL-6 and gp130 were assessed by cytoplasmic staining of plasma cells.
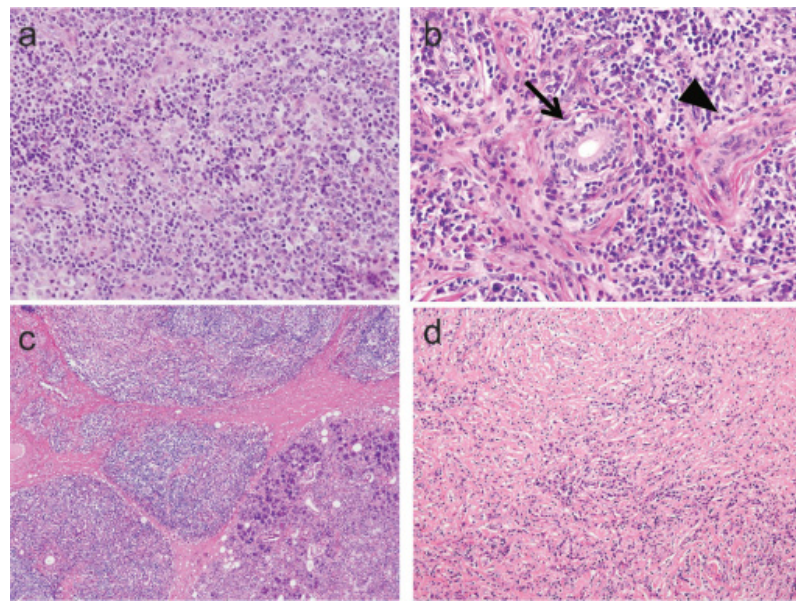

Figure 1a-d. Fibrosis grade. (a) The lymph node showing diffuse infiltration by lymphocytes and plasma cells without fibrosis, Grade 0.(b) The submandibular gland showing slight periductal (arrow) and perivascular fibrosis (arrow head), Grade 1. (c) The parenchyma of the submandibular gland is separated by thick collagen bundles, Grade 2. (d)The parenchyma of the submandibular gland is replaced by dense fibrosis,Grade 3. a-d, hematoxylin-eosin stain; a, b 200×; c 40×; d 100×.

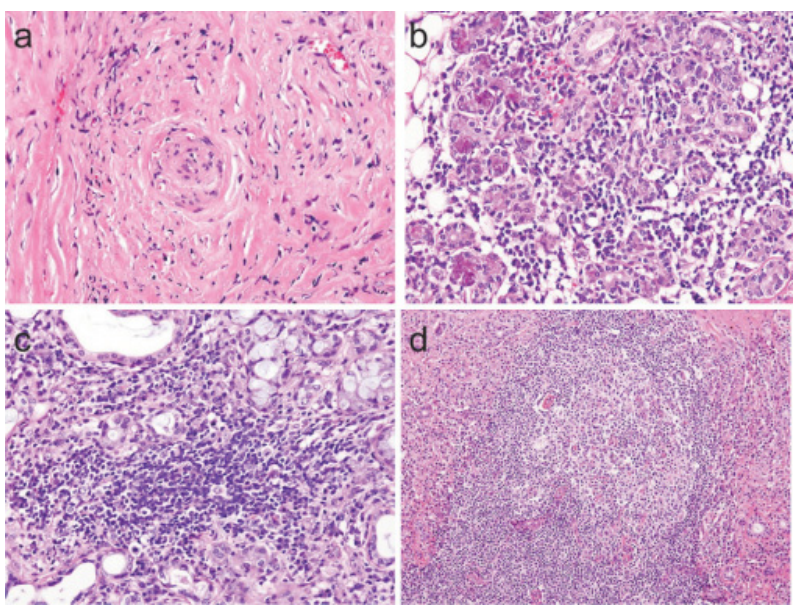

Figure 2a-d. Lymphocyte infiltration grade. (a) There is very slight infiltration of lymphocytes in the submandibular gland, Grade 0. (b)The pancreas showing mild infiltration of lymphocytes around the acinar cells, Grade 1. (c) Aggregation of lymphocytes without an agerminal center in the minor salivary gland, Grade 2. Acinar cells showing atrophic change. (d) The submandibular gland showing hyperplastic lymphoid follicle with a germinal center, Grade 3. a-d,hematoxylin-eosin stain; a, b, c 200×; d 40×

\section{Statistical analysis}

All statistical analyses were performed using PRISMver.6 (GraphPad Software, La Jolla, CA, USA).Comparisons were made using the Mann-Whitney Utest, and comparisons for a positive rate of SF, OP, IL-6, and gp-130 were made using chi-square test. The receiver operating characteristic curves were constructed and compared with area under the curve to determine the cut-off values for the positive rate of IL- 6 and gp130. P $<0.05$ was considered as statistically significant.

\section{Results}

\section{Age and sex}

The IgG4-RD cases were subsequently divided into the following three groups: definite IgG4-RD $(n=83)$, probable IgG4-RD $(n=5)$, and possible IgG4-RD ( $\mathrm{n}=6)$ according to CDC for IgG4-RD (Table 1). The male:female ratio was 62:32 among IgG4-RD cases and24:10 among non-IgG4-RD cases, and patients in the former group were older than those in the latter group $(63.0 \pm 12.1$ years vs $53.0 \pm 18.8$ years; $\mathrm{p}=0.0072$ ). Definite IgG4-RD, probable IgG4-RD, and non-IgG4-RD showed male predominance (Table 1). No patients under the age of 30 years were included in either group.

\section{Sites}

The distribution of the 177 lesions in the IgG4-RD group was as follows. The maximum number of involved organs were six (submandibular gland,parotid gland, lacrymal gland, lymph node, paranasal sinus and prostatic gland; submandibular gland,lacrymal gland, lymph node, lung, retroperitoneum, and ureter). The number of affected organs and cases was $5(\mathrm{n}=3), 4(\mathrm{n}=7), 3(\mathrm{n}=$ $14), 2(\mathrm{n}=12)$, and $1(\mathrm{n}=56)$. Exocrine glands, including major salivary glands $(31.1 \%)$, lacrimal gland $(16.4 \%)$, minor salivary glands $(6.8 \%)$, and pancreas $(6.8 \%)$, were affected in all IgG4-RD lesions. 
Table 1. Clinicopathological findings of IgG4-related disease and non-IgG4-related disease

\begin{tabular}{|c|c|c|c|c|}
\hline \multicolumn{5}{|c|}{ IgG4-RD ( $\mathrm{n}=94)$} \\
\hline & Definite $(\mathrm{n}=83)$ & Probable $(\mathrm{n}=5)$ & Possible $(n=6)$ & Non-IgG4-RD $(\mathrm{n}=34)$ \\
\hline Age (y/o) & $62.7 \pm 11.7^{* *}$ & $56.8 \pm 18.4$ & $71.5 \pm 6.7^{*}$ & $53.0 \pm 18.8$ \\
\hline $\operatorname{Sex}(M: F)$ & $57: 26$ & $3: 2$ & 2:4 & $24: 10$ \\
\hline S-IgG4 (mg/dL) & $903.9 \pm 649.4^{* * *}$ & $87.0 \pm 44.7^{\star}$ & $835.2 \pm 400.1$ & $418.8 \pm 504.9$ \\
\hline IgG4/HPF & $139.9 \pm 71.0^{* * *}$ & $79.2 \pm 49.5$ & $9.6 \pm 10.9^{* *}$ & $75.4 \pm 95.6$ \\
\hline Lymphocyte-infiltration grade & $2.2 \pm 0.8$ & $2.2 \pm 1.3$ & $1.1 \pm 1.1^{*}$ & $2.4 \pm 1.0$ \\
\hline Eosinophils/5 HPFs & $27.4 \pm 118.1^{*}$ & $101.0 \pm 190.0^{*}$ & $0.6 \pm 1.6$ & $14.2 \pm 42.4$ \\
\hline Mastcells/5 HPFs & $15.2 \pm 25.8^{*}$ & $93.8 \pm 183.5$ & $9.8 \pm 18.7$ & $30.0 \pm 47.3$ \\
\hline Fibrosis grade & $1.9 \pm 1.0^{* * * *}$ & $2.2 \pm 1.3^{*}$ & $1.7 \pm 1.0^{*}$ & $0.7 \pm 1.1$ \\
\hline Storiform fibrosis n (\%) & $22(26.5 \%)^{* *}$ & $1(20.0 \%)$ & $1(16.7 \%)$ & $1(2.5 \%)$ \\
\hline Obliterative phlebitis n (\%) & $27(32.5 \%)^{* * \star}$ & $2(40.0 \%)^{\star \star}$ & $1(16.7 \%)$ & $1(2.5 \%)$ \\
\hline
\end{tabular}

Abbreviations: $n$, number; ${ }^{*}, \mathrm{p}<0.05 ;{ }^{* *}, \mathrm{p}<0.01 ;{ }^{* * *}, \mathrm{p}<0.001{ }^{* * * *}, \mathrm{p}<0.0001$; IgG, immunoglobulin $\mathrm{G} ; \mathrm{RD}$, related disease; y/o, years old; M, male; F, female; $\mathrm{S}$, serum; HPF, high powered field

The distribution of the 37 lesions in the non-IgG4-RD group were as follows: $54.1 \%$ in the lymph nodes $(\mathrm{n}=20) ; 10.8 \%$ in the major salivary glands $(\mathrm{n}=4) ; 8.1 \%$ in the lung $(\mathrm{n}=3)$ and lachrymal gland $(n=3) ; 5.4 \%$ in the kidney $(n=2) ; 2.7 \%$ in the pancreas $(n=1)$, prostatic gland $(n=1)$, bone marrow $(n=1)$, skin $(\mathrm{n}=1)$, and retroperitoneum $(\mathrm{n}=1)$. The maximumnumber of involved organs was two; two cases ofMCD showed the involvement of two organs (lungand lymph node). Other 33 lesions in the non-IgG4-RD group affected a single organ.

\section{S-IgG4 level}

S-IgG4 was significantly higher in the IgG4-RDgroup (855.5 \pm $640.7 \mathrm{mg} / \mathrm{dL}, \mathrm{n}=94)$ than in the nonIgG4-RD group (418.8 \pm $504.9 \mathrm{mg} / \mathrm{dL}, \mathrm{n}=34)(\mathrm{p}=0.0013)$; definite IgG4-RD exhibited significantlyhigher levels than non-IgG4-RD $(903 \pm 649.4 \mathrm{mg} / \mathrm{dL}, \mathrm{p}$ $<0.001$ )(Table 1).

\section{Histological features}

Tissue specimens of definite IgG4-RD showed abundant lymphoplasmacytic infiltration (Fig. 3a andFig. 3b), SF, and OP (Fig. 3c and Fig. 3d). DefiniteIgG4-RD also had dense fibrosis and septal fibrosis accompanied by SF. At the periphery of the lesions,periductal fibrosis and perivascular fibrosis were observed. Acinar glands were surrounded by fine fibrosis, particularly in the case of the pancreas, salivary glands, and lacrymal gland. Immunohistochemically,many of the infiltrating plasma cells were positive for IgG and IgG4 (Fig. 3e and Fig. 3f). Compared with definite IgG4-RD, probable IgG4-RD showed noticeably decrease of IgG4-positive plasma cell infiltration, an increase of eosinophil and mast cell infiltration, and marked fibrosis. Possible IgG4-RD exhibited slight inflammatory cell infiltration (10 >lymphocytes and plasma cells/HPF)(Table 1).

The non-IgG4-RD cases completely or partially matched CDC guidelines for IgG4-RD. Some of the non-IgG4-RD cases could be easy differentiated from the others based on the histological findings only; however, some cases exhibited similar clinical and histological findings to IgG4-RD. In particular, it was difficult to distinguish MCD from IgG4-RD because of their histological similarity, a final diagnosis was reached based on clinical information.

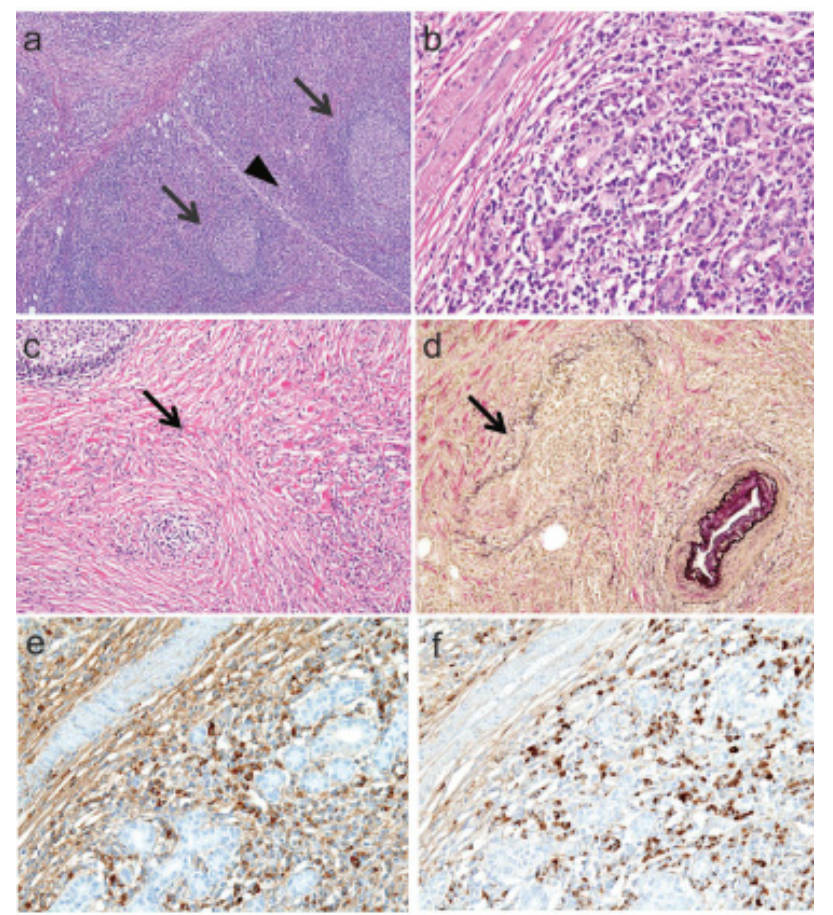

Figure 3a-f. Pathological findings. (a) Indefinite IgG4-related disease, chronic inflammatory cells markedly infiltrate into the submandibular glands. Hyperplastic lymphoid follicles are present (arrows), and fibrosclerotic changes are seen in the interfollicular area (arrow head). (b)Interstitial infiltration of lymphocytes and plasma cells is seen in the submandibular gland. (c) Scattered lymphocytes and plasma cells are seen in the dense fibrotic area. Collagen fibers exhibit storiform fibrosis(arrow). (d) Chronic inflammatory cells infiltrate into the venous walls and form obliterative phlebitis (arrow). (e) Dense infiltration of IgGpositive plasma cells is seen. (f) Many of the IgG-positive plasma cells are positive for IgG4. a-c, hematoxylin-eosin stain; d, elastic vanGieson stain, e, IgG; f, IgG4; a $40 \times$; b, e, f 200×; c, d 100×.

\section{IgG4/IgG ratios and IgG4/HPF}

IgG4/IgG ratios were the highest indefinite IgG4-RD and were significantly different between definiteIgG4-RD ( $\mathrm{p}<0.0001)$, 
probable IgG4-RD ( $\mathrm{p}<0.01)$, possible IgG4-RD $(\mathrm{p}<0.05)$, and non-IgG4-RD(Table 1). In the IgG4-RD groups, definite IgG4-RD showed the highest mean number of IgG4/HPF, with a statistically significant difference (Table 1).

Table 2 summarizes the average of the $\mathrm{IgG} 4 / \mathrm{IgGratios}$ and $\mathrm{IgG} 4 / \mathrm{HPF}$ in each of the organs or tissues.

Table 2. Average of IgG4/IgG ratios and IgG4/HPF of IgG4-relateddisease in each site

\begin{tabular}{|c|c|c|c|c|c|c|}
\hline \multicolumn{5}{|c|}{$\operatorname{IgG} 4 / \operatorname{IgG} \%(\mathrm{n})$} & \multicolumn{2}{|c|}{$\operatorname{IgG} 4 / \mathrm{HPF}(\mathrm{n})$} \\
\hline Definite & & Probable & Possible & Definite & Probable & Possible \\
\hline Major salivary gland $(\mathrm{n}=34)$ & $76.8(29)$ & $74.8(2)$ & $13.8(3)$ & $154.8(29)$ & $66.5(2)$ & $9.9(3)$ \\
\hline Lacrymal gland $(\mathrm{n}=17)$ & $77.3(16)$ & $74.7(1)$ & & $135.0(16)$ & $52.6(1)$ & \\
\hline Minor salivary gland $(\mathrm{n}=12)$ & $83.1(10)$ & & $16.4(2)$ & $112.7(10)$ & & $13.6(2)$ \\
\hline Kidney $(\mathrm{n}=10)$ & $70.4(10)$ & & & $139.7(10)$ & & \\
\hline $\operatorname{Pancreas}(\mathrm{n}=3$ & $73.6(3)$ & & & $47.1(3)$ & & \\
\hline $\operatorname{Lung}(\mathrm{n}=3)$ & $80.1(3)$ & & & $151.9(3)$ & & \\
\hline Lymph node $(\mathrm{n}=3)$ & $83.8(3)$ & & & $166.3(3)$ & & \\
\hline Retroperitoneum $(\mathrm{n}=3)$ & $92.8(2)$ & $44.1(1)$ & & $99.9(2)$ & $44.8(1)$ & \\
\hline Intestine $(\mathrm{n}=2)$ & $91.5(1)$ & & $4.0(1)$ & $136.0(1)$ & & $0.8(1)$ \\
\hline Bile $\operatorname{duct}(\mathrm{n}=1)$ & $48.6(1)$ & & & $92.6(1)$ & & \\
\hline Mediastinum $(\mathrm{n}=1)$ & $85.1(1)$ & & & $37.8(1)$ & & \\
\hline $\operatorname{Liver}(\mathrm{n}=1)$ & $74.1(1)$ & & & $77.8(1)$ & & \\
\hline Prostatic gland $(\mathrm{n}=1)$ & $100.0(1)$ & & & $170.2(1)$ & & \\
\hline Paranasal sinus $(\mathrm{n}=1)$ & $66.6(1)$ & & & $114.6(1)$ & & \\
\hline $\operatorname{Pericardium}(\mathrm{n}=1)$ & $86.9(1)$ & & & $37.8(1)$ & & \\
\hline $\operatorname{Skin}(\mathrm{n}=1)$ & & $51.2(1)$ & & & $165.6(1)$ & \\
\hline Total average & $79.4(83)$ & $61.2(5)$ & $11.4(6)$ & $111.6(83)$ & $82.4(5)$ & $8.1(6)$ \\
\hline
\end{tabular}

Abbreviations: IgG, immunoglobulin G; HPF, high powered field; $n$, number

The average of the IgG4/IgG ratios in each of the sites were $79.4 \%$ in the definite IgG4-RD group, $61.2 \%$ inthe probable IgG4-RD group (range: $48.6 \%-100 \%$ and $44.1 \%-74.8 \%$,respectively) and $11.4 \%$ in the possible IgG4-RD group (range: $4.0 \%-16.4 \%$ ). The average number of IgG4/HPF in each of the sites was111.6 in the definite IgG4-RD group (range: $37.8-166.3$ ) and 82.4 in the probable IgG4-RD group(range: 44.8-165.6). The average of possible IgG4-RD was equal to 8.1 (range: 0.8-13.6). The IgG4/IgGrations of retroperitoneum, bile duct, and skin indicated lower levels. The IgG4/HPF of pancreas,retroperitoneum, mediastinum, and pericardium indicated lower levels. No significant differences in $\operatorname{IgG} 4 / \mathrm{IgG}$ ratios and $\operatorname{IgG} 4 / \mathrm{HPF}$ were observedbetween organs.

The average of the IgG4/IgG ratios in each of the siteswere $79.4 \%$ in the definite IgG4-RD group, $61.2 \%$ in the probable IgG4-RD group (range: $48.6 \%-100 \%$ and $44.1 \%-74.8 \%$, respectively) and $11.4 \%$ in thepossible IgG4-RD group (range: $4.0 \%-16.4 \%$ ). The average number of IgG4/HPF in each of the sites was111.6 in the definite IgG4-RD group (range: $37.8-166.3$ ) and 82.4 in the probable IgG4-RD group(range: 44.8-165.6). The average of possible IgG4-RD was equal to 8.1 (range: 0.8-13.6). The IgG4/IgGrations of retroperitoneum, bile duct, and skin indicated lower levels. The IgG4/HPF of pancreas,retroperitoneum, mediastinum, and pericardium indicated lower levels. No significant differences in IgG4/IgG ratios and IgG4/HPF were observed between organs.

The averages of the IgG4/IgG ratios and IgG4/HPF in the non-IgG4-RD group are summarized in Table 3. IgG4/IgG ratios in MCD, malignant lymphoma (MALToma), Crow-Fukase disease,
andKimura's disease were $>40 \%$. Average of IgG4/HPFin non-IgG4-RD exceeded $>10$, and an increase in IgG4-positive plasma cells was particularly noticeable in MCD cases.

Table 3. Average of IgG4/IgG ratios and IgG4/HPF in non-IgG4related disease

\begin{tabular}{ccc}
\hline & IgG4/IgG ratio(\%) & IgG4/HPF \\
\hline Castleman's disease(14) & $29.4 \pm 21.7$ & $116.6 \pm 130.3$ \\
Multicentric(6) & $41.2 \pm 21.7$ & $181.7 \pm 130.3$ \\
Unicentric(8) & $20.5 \pm 16.4$ & $67.9 \pm 72.5$ \\
Malignant lymphoma(7) & $44.5 \pm 31.5$ & $64.2 \pm 68.2$ \\
MALToma(6) & $47.6 \pm 31.5$ & $65.6 \pm 68.2$ \\
AILT(1) & 26.0 & 55.8 \\
NSRL(3) & $18.4 \pm 15.6$ & $46.4 \pm 45.5$ \\
Chronic sialoadenitis(2) & $12.7 \pm 17.9$ & $13.3 \pm 18.8$ \\
Warthin's tumor(2) & $28.3 \pm 3.0$ & $37.0 \pm 36.2$ \\
Crow-Fukase disease(1) & 45.7 & 34.0 \\
Kimura’s disease(1) & 40.9 & 64.4 \\
Interstitial nephritis(1) & 32.6 & 22.8 \\
Membranous nephropathy(1) & 17.3 & 26.0 \\
Retroperitoneal fibrosis(1) & 24.5 & 27.8 \\
IMT(1) & 24.6 & 67.0 \\
\hline
\end{tabular}

mucosa associated lymphoid tissue; AILT, angioimmunoblastic T-cell lymphoma; NSRL, non-specific reactive lymphadenopathy; IMT, inflammatorymyofibroblastic tumor 


\section{Lymphocyte-infiltration grade and infiltration of eosinophils and mast cells}

There were no significant differences in lymphocyte -infiltration grade among definite IgG4-RD, probable IgG4-RD, and non-IgG4- RD groups; the lymphocyte-infiltration grade was the lowest impossible IgG4-RD (Table 1). Lymphocyte- infiltration grade of minor salivary glands and lymph nodes were higher than those of other organs (Table 4). Significant differences in the number of eosinophils were observed among definite IgG4-RD $(27.4 \pm 118.1, \mathrm{p}<0.05)$, probable IgG4-RD $(101.0 \pm 190.0, \mathrm{p}<$ $0.05)$, and non-IgG-RD groups. Significant differences in the number of mast cells were observed between definite IgG4-RD $(15.2 \pm 25.8, \mathrm{p}<0.05)$ and nonIgG-RD groups. The number of eosinophils and mast cells in probable IgG4-RD indicated the highest levels(Table 1).

Table 4. Average of lymphocyte-infiltration grade and fibrosis grade ofIgG4-related disease in each site

\begin{tabular}{|c|c|c|c|c|c|c|}
\hline \multicolumn{5}{|c|}{ Lymphocyte-infiltration grade(n) } & \multicolumn{2}{|c|}{ Fibrosis grade(n) } \\
\hline Definite & & Probable & Possible & Definite & Probable & Possible \\
\hline Major salivary gland $(\mathrm{n}=34)$ & $2.7(29)$ & $2.5(2)$ & $1.7(3)$ & $2.5(29)$ & $3.0(2)$ & $2.3(3)$ \\
\hline Lacrymal gland $(\mathrm{n}=17)$ & $2.4(16)$ & $3.0(1)$ & & $1.4(16)$ & $0.0(1)$ & \\
\hline Minor salivary gland $(\mathrm{n}=12)$ & $1.8(10)$ & & $2.0(2)$ & $1.5(10)$ & & $1.5(2)$ \\
\hline Kidney $(\mathrm{n}=10)$ & $1.3(10)$ & & & $1.5(10)$ & & \\
\hline Pancreas $(\mathrm{n}=3$ & $1.7(3)$ & & & $3.0(3)$ & & \\
\hline $\operatorname{Lung}(\mathrm{n}=3)$ & $2.3(3)$ & & & $1.3(3)$ & & \\
\hline Lymph node $(\mathrm{n}=3)$ & $3.0(3)$ & & & $0.0(3)$ & & \\
\hline Retroperitoneum(n = 3) & $1.5(2)$ & $0.0(1)$ & & $3.0(2)$ & $3.0(1)$ & \\
\hline Intestine $(\mathrm{n}=2)$ & $2.0(1)$ & & $0.0(1)$ & $0.0(1)$ & & $0.0(1)$ \\
\hline Bile $\operatorname{duct}(\mathrm{n}=1)$ & $1.0(1)$ & & & $3.0(1)$ & & \\
\hline Mediastinum $(\mathrm{n}=1)$ & $1.0(1)$ & & & $1.0(1)$ & & \\
\hline $\operatorname{Liver}(\mathrm{n}=1)$ & $1.0(1)$ & & & $3.0(1)$ & & \\
\hline Prostatic gland $(\mathrm{n}=1)$ & $2.0(1)$ & & & $1.0(1)$ & & \\
\hline Paranasal sinus $(\mathrm{n}=1)$ & $1.0(1)$ & & & $3.0(1)$ & & \\
\hline $\operatorname{Pericardium}(\mathrm{n}=1)$ & $1.0(1)$ & & & $3.0(1)$ & & \\
\hline $\operatorname{Skin}(\mathrm{n}=1)$ & & $3.0(1)$ & & & $2.0(1)$ & \\
\hline Total average & $1.7(83)$ & $2.2(5)$ & $1.2(6)$ & $1.9(83)$ & $2.2(5)$ & $1.3(6))$ \\
\hline
\end{tabular}

Abbreviation: $\mathrm{n}$, number

\section{Fibrosis grade}

The fibrosis grade was significantly lower $(0.7 \pm 1.1)$ in the non-IgG4-RD group than in thedefiniteIgG4-RD $(1.9 \pm 1.0, \mathrm{p}<$ $0.0001)$, probable $\operatorname{IgG} 4-\mathrm{RD}(2.2 \pm 1.3, \mathrm{p}<0.05)$, and possible IgG4-RD $(1.7 \pm 1.0, \mathrm{p}<0.05)$. Fibrosis grade was the highest improbable IgG4-RD (Table 1)

The incidence of SF and OP were seen to gradually increase with fibrosis grade (Fig. 4a andFig. 4b). The fibrosis grade of the major salivary glands, pancreas, retroperitoneum, bile duct, liver, paranasal sinus, and pericardium were higher than that of other organs (Table 4).

\section{SF and OP}

SF was observed in 22 cases $(26.5 \%, \mathrm{p}<0.01)$ of definite IgG4-RD but was in probable IgG4-RD (20.0\%), possible IgG4-RD (16.7\%), and non-IgG4-RD (2.5\%)(Table 1). OP was observed in 27 cases of definite IgG4-RD (32.5\%, p < 0.001). It was observed in two cases of probable IgG4-RD $(40.0 \%, \mathrm{p}<0.01)$, one case of possible IgG4-RD (16.7\%), and one case of non-IgG4-RD (2.5\%) groups (Table 1).

High incidence of SF and OP was observed in the major salivary glands, pancreas, and retroperitoneum(Table 5). SF and OP were seen in one of 6 case ofMCD.
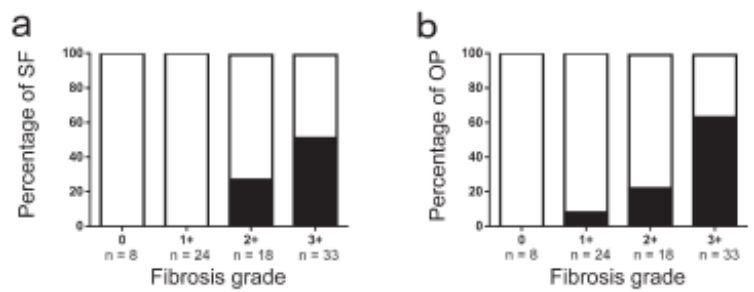

Figure 4a, b. Correlation between fibrosis grade and lymphocyte infiltration grade. Storiform fibrosis and obliterative phlebitis gradually increased with fibrosis grade.Abbreviations: n, number; SF, storiform fibrosis; OP, obliterative phlebitis.

\section{Biopsied and surgically resected specimens}

There were no apparent differences in IgG4/IgGratios and IgG4/HPFs between biopsied and surgically-resected specimens; biopsied specimens of definiteIgG4-RD exhibited significantly a lower fibrosis grade, lymphocyte-infiltration grade, SF, and OP compared with surgically resected specimens (Table6). All cases of probable IgG4-RD were surgically-resected specimens; all cases of possible IgG4-RDwere biopsied specimens. 
Table 5. Average of storiform fibrosis and obliterative phlebitis oflgG4-related disease in each site

\begin{tabular}{|c|c|c|c|c|c|c|}
\hline & \multicolumn{3}{|c|}{$\mathrm{SF} \%(\mathrm{n})$} & \multicolumn{3}{|c|}{$\mathrm{OP} \%(\mathrm{n})$} \\
\hline & Definite & Probable & Possible & Definite & Probable & Possible \\
\hline Majorsalivary gland( $\mathrm{n}=34)$ & $42.9(29)$ & $0.0(2)$ & $33.3(3)$ & $50.0(29)$ & $50.0(2)$ & $33.3(3)$ \\
\hline Lacrymal gland $(\mathrm{n}=17)$ & $6.2(16)$ & $0.0(1)$ & & $18.8(16)$ & $0.0(1)$ & \\
\hline Minor salivary gland $(\mathrm{n}=12)$ & $0.0(10)$ & & $0.0(2)$ & $0.0(10)$ & & $0.0(2)$ \\
\hline Kidney $(\mathrm{n}=10)$ & $20.0(10)$ & & & $20.0(10)$ & & \\
\hline Pancreas $(\mathrm{n}=3)$ & $33.3(3)$ & & & $66.7(3)$ & & \\
\hline $\operatorname{Lung}(\mathrm{n}=3)$ & $33.3(3)$ & & & $33.3(3)$ & & \\
\hline Lymph node $(\mathrm{n}=3)$ & $0.0(3)$ & & & $0.0(3)$ & & \\
\hline Retroperitoneum(n = 3) & $50.0(2)$ & $0.0(1)$ & & $50.0(2)$ & $100.0(1)$ & \\
\hline Intestine $(\mathrm{n}=2)$ & $0.0(1)$ & & $0.0(1)$ & $0.0(1)$ & & $0.0(1)$ \\
\hline Bile $\operatorname{duct}(\mathrm{n}=1)$ & $100.0(1)$ & & & $100.0(1)$ & & \\
\hline Mediastinum $(\mathrm{n}=1)$ & $0.0(1)$ & & & $0.0(1)$ & & \\
\hline $\operatorname{Liver}(\mathrm{n}=1)$ & $100.0(1)$ & & & $0.0(1)$ & & \\
\hline Prostatic gland $(\mathrm{n}=1)$ & $0.0(1)$ & & & $0.0(1)$ & & \\
\hline Paranasal sinus $(\mathrm{n}=1)$ & $100.0(1)$ & & & $100.0(1)$ & & \\
\hline $\operatorname{Pericardium}(\mathrm{n}=1)$ & $100.0(1)$ & & & $100.0(1)$ & & \\
\hline $\operatorname{Skin}(\mathrm{n}=1)$ & & $100.0(1)$ & & & $0.0(1)$ & \\
\hline Total average & $39.0(83)$ & $20.0(5)$ & $11.1(6)$ & $35.9(83)$ & $40.0(5)$ & $11.1(6)$ \\
\hline
\end{tabular}

Abbreviations: SF, storiform fibrosis; OP, obliterative phlebitis; n, number

Table 6. Clinicopathological findings of definite IgG4-related disease in biopsied and surgically resected specimens

\begin{tabular}{cccc}
\hline & & DefiniteIgG4-RD $(\mathrm{n}=83)$ & \\
\cline { 2 - 4 } & Biopsy $(\mathrm{n}=63)$ & Surgical $(\mathrm{n}=20)$ & $\mathrm{p}$ \\
\hline Age (y/o) & $61.9 \pm 1.4$ & $65.1 \pm 2.6$ & $\mathrm{NS}$ \\
Sex (M : F) & $45: 18$ & $12: 8$ & $\mathrm{NS}$ \\
S-IgG4 (mg/dL) & $920.9 \pm 83.4$ & $926.5 \pm 229.0$ & $\mathrm{NS}$ \\
IgG4/IgG ratrios (\%) & $76.1 \pm 1.8$ & $82.5 \pm 3.2$ & $\mathrm{NS}$ \\
IgG4/HPF & $129.5 \pm 9.2$ & $147.5 \pm 4.1$ & 0.0203 \\
Lymphocyte-infiltration grade & $2.0 \pm 0.1$ & $2.5 \pm 0.1$ & $\mathrm{NS}$ \\
Eosinophils/5 HPFs & $32.4 \pm 17.7$ & $12.6 \pm 3.6$ & $\mathrm{NS}$ \\
Mast cells/5 HPFs & $15.3 \pm 3.8$ & $14.9 \pm 6.5$ & 0.0033 \\
Fibrosis grade & $1.7 \pm 0.1$ & $2.5 \pm 0.1$ & 0.0315 \\
Storiform fibrosis n (\%) & $13(20.6 \%$ & $9(45.0 \%)$ & 0.0138 \\
Obliterative phlebitis n (\%) & $16(19.3 \%)$ & $11(55.0 \%)$ & \\
\hline
\end{tabular}

Abbreviations: IgG, immunoglobulin G; RD, related disease; $n$, number; NS, not significant; y/o, years old; M, male; F, female; S, serum; HPF, high powered field

\section{Single or multiple organ involvement}

Indefinite IgG4-RD, there were no significant differences in all parameters between cases involving a single organ $(\mathrm{n}=51)$ and those involving multiple organs $(n=32)$ (Table 7$)$. However, S-IgG4 levels of cases involving multiple organs tended to be higher than those of cases involving a single organ $(1,085 \pm 115.5 \mathrm{mg} / \mathrm{dL}$ vs. $820.3 \pm 112.9 \mathrm{mg} / \mathrm{dL}, \mathrm{p}=0.6126)$.

All 5 cases of probable IgG4-RD exhibited single organ involvement; all 6 cases of possible IgG4-RDshowed multiple organ involvement.

\section{Relapse after steroid therapy}

Improved S-IgG4, S-IgG, and serum IgG4/IgGratios (S-IgG4/IgG ratios) and disappearance of organ enlargement were observed in 15 cases of definiteIgG4-RD subjected to steroid therapy. The mean duration of steroid therapy was 45.4 months (range,19-84 months), and the average dose was $33 \mathrm{mg} /$ day(range, $20-45 \mathrm{mg} /$ day). However, 6 of 15 cases had prolonged S-IgG4 elevation, which gradually increased after the steroid therapy, and 3 of 6 cases exhibited submandibular gland and lacrimal gland re-enlargement (average, 20.7 months; range, 7-47 months). S-IgG4 and S-IgG levels were decreased significantly after the steroid therapy in nine 
Table 7. Clinicopathological findings between cases involving a singleorgan and multiple organs in definite IgG4-related disease

\begin{tabular}{ccc}
\hline & \multicolumn{2}{c}{ DefinitelgG4-RD(n=83) } \\
\cline { 2 - 3 } & Single organ $(\mathrm{n}=51)$ & Multiple organs $(\mathrm{n}=32)$ \\
\hline Age (y/o) & $62.8 \pm 1.9$ & $62.6 \pm 1.7$ \\
Sex (M : F) & $34: 17$ & $23: 9$ \\
S-IgG4 (mg/dL) & $820.3 \pm 112.9$ & $1,085.0 \pm 115.5$ \\
IgG4/IgG ratrios (\%) & $77.3 \pm 13.8$ & $78.4 \pm 16.7$ \\
IgG4/HPF & $126.8 \pm 68.5$ & $145.1 \pm 74.5$ \\
Lymphocyte-infiltration grade & $2.2 \pm 0.1$ & $2.1 \pm 0.2$ \\
Eosinophils/5 HPFs & $36.7 \pm 22.1$ & $13.8 \pm 5.0$ \\
Mast cells/5 HPFs & $15.8 \pm 4.3$ & $14.5 \pm 5.2$ \\
Fibrosis grade & $2.1 \pm 0.1$ & $1.7 \pm 0.2$ \\
Storiform fibrosis n (\%) & $10(19.6 \%)$ & $12(37.5 \%)$ \\
Obliterative phlebitis n (\%) & $15(29.4 \%)$ & $12(37.5 \%)$
\end{tabular}

Abbreviations: IgG, immunoglobulin G; RD, related disease; $n$, number; $y / o$, years old; $M$, male; F, female; $S$, serum; HPF, high powered field

nonrelapsed cases (Table $8, \mathrm{p}<0.0001$, and $\mathrm{p}<0.001$ ). Six relapsed cases also showed decreased S-IgG4 and S-IgG without significant differences 1 year after steroid therapy (Table 8). Nine non-relapsed cases included 6 cases involving a single organ and 3 cases involving multiple organs; six relapsed cases included 1 case with single organ involvement and 5 cases with multiple organ involvement. Among the non-relapsed cases, SF was found in 3 of 9 cases $(33.3 \%)$ and OP was found in 4 of 9 cases $(44.4 \%)$. SF and OP were not found in relapsed cases. IgG4/IgG ratios,IgG4/HPF, eosinophils/5 HPFs, mast cells/5 HPFs,fibrosis grade, and lymphocyte-infiltration grade in the non-relapsed cases were not significantly different from the relapsed cases.

Table 8. Serum IgG, IgG4 and their ratios before and after steroid therapy for non-relapsed and relapsed definite IgG4-related disease

\begin{tabular}{ccccc}
\hline & \multicolumn{2}{c}{ Non-relapsed(n=9) } & \multicolumn{2}{c}{ Relapsed(n=6) } \\
\cline { 2 - 5 } & Before & After & Before & After \\
\hline S-IgG(mg/dL) & $2,427.4^{* * * *}$ & 991.4 & $1,883.3$ & $1,385.7$ \\
S-IgG4(mg/dL) & $917.4^{* * *}$ & 184.0 & 649.5 & 393.0 \\
S-IgG4/IgG ratios(\%) & 35.0 & 19.3 & 33.3 & 25.9 \\
\hline
\end{tabular}

Abbreviations: $n$, number; ${ }^{* * *}, p<0.001 ;{ }^{* * *}, p<0.0001 ;$ Before, before steroid therapy; After, 1 year after steroid therapy; $S$, serum; HPF, high powered field

\section{Clinicopathological and immunohistochemical comparison} of definite IgG4-RD and MCD

Age, IgG4/IgG4 ratios, and fibrosis grade were significantly higher in definite IgG4-RD cases when compared with the MCD cases (Table 9).

The threshold for IL- 6 and gp130 was selected to be more than $20 \%$ of the positive rates for plasma cells based on the best diagnostic efficacy achieved through equilibrium between sensitivity and specificity. The immunohistochemical positivity for IL-6 was $21.4 \%$ indefinite IgG4-RD cases $(n=70)$ and $83.3 \%$ in MCDcases $(n=6)$. A significant difference was observed between definite IgG4-RD and MCD ( $\mathrm{p}=0.001)$.Gp130 showed a higher positive rate for both definiteIgG4-RD $(79.2 \%, \mathrm{n}=48)$ and MCD $(100.0 \%, \mathrm{n}=5)$, but this difference was not statistically significant ( $p=0.2572)$ (Fig. 5a, and Fig. 5b).

Table 9. Difference of clinicopathological findings between definiteIgG4-related disease and multicentric Castleman's disease

\begin{tabular}{ccc}
\hline & Definite IgG4-RD(n=83) & MCD $(\mathrm{n}=6)$ \\
\hline Age (y/o) & $62.7 \pm 11.7^{* *}$ & $47.0 \pm 14.6$ \\
Sex (M : F) & $57: 26$ & $5: 1$ \\
S-IgG4 (mg/dL) & $903.9 \pm 649.4$ & $349.8 \pm 120.0$ \\
IgG4/IgG ratrios (\%) & $77.7 \pm 14.8^{* * *}$ & $41.2 \pm 23.5$ \\
IgG4/HPF & $139.9 \pm 71.0$ & $181.7 \pm 167.1$ \\
Lymphocyte-infiltration grade & $2.2 \pm 0.8$ & $2.7 \pm 0.8$ \\
Eosinophils/5 HPFs & $27.4 \pm 118.1$ & $8.5 \pm 14.2$ \\
Mast cells/5 HPFs & $15.2 \pm 25.8$ & $50.6 \pm 77.8$ \\
Fibrosis grade & $1.9 \pm 1.0^{* *}$ & $0.7 \pm 1.2$ \\
Storiform fibrosis n (\%) & $22(26.5 \%)$ & $1(16.7 \%)$ \\
Obliterative phlebitis n (\%) & $27(32.5 \%)$ & $1(16.7 \%)$ \\
\hline
\end{tabular}

Abbreviations: IgG, immunoglobulin G; RD, related disease; n, number; MCD, multicentric Castleman's disease; y/o, years old; $M$, male; F, female; $S$, serum; HPF, high powered field 


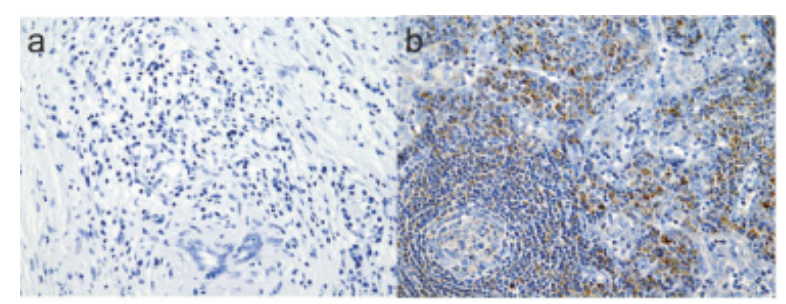

Figure 5a, b. Immunohistochemical findings for interleukin-6. (a) Plasma cells are not staining in the case of definite IgG4-related disease of the pancreas. (b) In the interfollicular area, plasma cells are positive in the case of Castleman's disease of the lymph node. a, b IL-6; a, b $200 \times$.

\section{Discussion}

CDC is the most pragmatic diagnostic criteria forIgG4-RD so far. However, clinicopathological features of probable and possible IgG4-RD using CDC has not yet been fully understood. Furthermore, other various diagnostic problems to be solved of CDC for IgG4-RD were also evaluated.

Probable IgG4-RD was characterized by IgG4/IgGratios $>40 \%$, $>10$ IgG4/HPF (but lower than definiteIgG4-RD), lower levels of S-IgG4, marked fibrosis, and increased numbers of eosinophil and mast cell infiltration. Masaki et al. ${ }^{12}$ reported that IgG4/HPFin the fibrotic parts of IgG4-RD was lower than in the non-fibrotic parts. The lower levels of S-IgG4 improbable IgG4-RD may be related to marked fibrosis and decreased IgG4/HPF. Eosinophils and mast cells are also known to be the major effector cells for tissue fibrosis and remodeling. ${ }^{13}$ They release various cytokines, including TGF- $\beta$, IL-13, IL-14, and vascular endothelial growth factor, which regulate fibroblastic function. ${ }^{13}$ The histological findings of probable IgG4-RD were similar to those of definiteIgG4-RD, except for the presence of marked fibrosis,decreased number of plasma cells, and increased number of eosinophils and mast cells. Probable IgG4-RD may be considered as the advanced phase of definite IgG4-RD.Possible IgG4-RD cases showed high S-IgG4, whereas the IgG4/IgG and IgG4/HPF ratios were lower. Additionally, marked fibrosis, lymphoplasmacytic infiltration, SF, and OP was notobserved. Although Umehara et al and Okazaki et $\mathrm{al}^{3,4}$ have recommended the application of organ-specific criteria for IgG4-RD, a repetition of the biopsy or surgical excision of other organs should be considered in such cases.

In the biopsied specimens, the IgG4/IgG ratios and IgG4/HPF of various organs and tissues could be evaluated. Compared with the surgically resected specimens, biopsied specimens showed significantly lower fibrosis grade and lymphocyte-infiltration grade, SF, and OP. Therefore, it is considered that the CDC is applied to a biopsied specimen as well as a surgically resected specimen. However, the former is smaller and does not reflect the entirety of the lesion,thus increasing the tendency for underestimation of the pathological diagnosis.

The number of affected organs and tissues differed between the IgG4-RD and non-IgG4-RD cases. TheIgG4-RD cases revealed multiple organs involvement when compared with the non-IgG4RD cases; in addition, selective exocrine organ involvement was also noted. Fibrosis grade is useful for the quantitative histological evaluation of IgG4-RD occurring invariable sites and tissues. As shown in Tables 4 and 5,deep-seated organs exhibiting higher fibrosis grades tended to show an increased incidence of SF and OP,suggesting that fibrosis is associated with the occurrence of SF and OP. The reason for this phenomenon is thought to be related to the anatomical site not easily noticed organ enlargement.

Steroid therapy for IgG4-RD has been widely recognized as a treatment measure, and it seems to be increasing opportunities to diagnose treated IgG4-RDcases. This study identified S-IgG4, S-IgG, S-IgG4/IgG ratios, number of involved organs, SF, and OP as good indicators for the prediction of the relapse of definite IgG4-RD after steroid therapy. Mizushima et $\mathrm{al}^{14}$ reported clinical and histological changes associated with steroid therapy in IgG4-relatedtubulointerstitial nephritis. They also reported that a decrease in IgG4-positive plasma cells was observed after steroid therapy, although interstitial fibrosis and small lymphocytic infiltration did not improve.Therefore, new therapeutic strategies for IgG4-RD is required by using these prediction factors.

The pathological features of MCD were very similar to those of IgG4-RD. However, useful

an immunohistochemical tool to distinguish MCD fromIgG4-RD has not yet known. Therefore, the final diagnosis of MCD had been based on clinical information. ${ }^{5} \mathrm{MCD}$ is known to exhibit overproduction of IL-6, leading to IgG4-positive plasma cell infiltration and serum IgG4 elevation. In this study, the positive expression of IL-6 in definiteIgG4-RD cases was significantly lower than that inMCD; the production of IL- 6 may be decreased inIgG4-RD patients. Hence, IL-6 immunostaining maybe useful as a diagnostic tool to distinguish betweenIgG4-RD and MCD.

Nakajima et al..$^{15}$ reported that impaired expression of innate immune response-related genes(including defensin) was associated with the development of IgG4-RD. Defensin families play an important role in the innate immune response against bacteria, fungi, protozoa, and viruses. Conversely, Ahn et $\mathrm{al}^{16}$ reported that defensin- 1 induces the secretion of IL- 6 in rheumatoid arthritis. IL-6 is one of the cytokines that control humoral immunity and is produced by various cells, including B-cells, T-cells,fibroblasts, monocytes, endothelial cells, and mesangial cells. ${ }^{17}$ It induces the differentiation of B-cells to plasma cells and naïve T-helper cells toTh17 cells; it also inhibits the differentiation of Treg-induced by TGF- $\beta$, and regulates the balance betweenTh17 and Treg immune reactions. ${ }^{18}$ Th17 plays a role in the defense against bacterial infections by enhancing neutrophil migration, whereas Treg is known to produce regulatory cytokines, such as IL-10and TGF- $\beta$, and facilitate IgG4 production, as well as fibrosis. ${ }^{19}$ Therefore, reduced expression ofneutrophil -derived innate immune response related genes (including defensin) may decrease IL-6secretion in IgG4-RD patients, leading to Tregdominant immune responses that may disturb the Treg/Th17 balance. ${ }^{18}$

In conclusion, the clinicopathological features oflgG4-RD and non-IgG4-RD occurring in various organs and tissues were analyzed. The diagnostic problems of CDC in IgG4-RD, the prediction oftherapeutic responsible factors, and the usefulness of IL-6 immunostaining for MCD were also discussed.However, the number of cases in the present study is limited, warranting the need for further studies to clarify the clinicopathological features of IgG4-RD and non-IgG4-RD.

This work was partially supported by a Grant-inAid for Scientific Research (C) of the Ministry of Culture and Science of Japan (25460465) and Health and Labour Sciences Research grants for IntractableDiseases from the Minister of Labour and Welfare ofJapan. The author would like to thank Drs. Hiroshi Minato, Eriko Kinoshita-Kobayashi, Satoko Nakada,Takayuki Nojima, Yasufumi Masaki, and HisanoriUmehara for their expert assistance and advice. The author would also like to thank the members 
ofMHLW for providing the cases and clinical data.

\section{Acknowledgments}

We thank Lee Seaman of Seaman Medical, Inc. (Bellingham, WA, USA) for providing professional English-language editing of this article.

\section{Conflict of interest statement}

The authors declare they have no conflicts of interest relevant to this manuscript.

\section{Funding}

This research did not receive any specific grant from funding agencies in the public, commercial, or not-for-profit sectors.

\section{Ethical approval}

This article does not contain any studies with human participants or animals performed by any of the authors.

\section{REFERENCES}

1. Umehara $\mathrm{H}$, Okazaki $\mathrm{K}$, Masaki $\mathrm{Y}$, et al: A novel clinical entity, IgG4-related disease(IgG4RD): general concept and details. Mod Rheumatol 2012; 22:1-14.

2. Deshpande V, Zen Y, Chan JK, et al. Consensus statement on the pathology of IgG4-related disease. Mod Pathol 2012; 25: 1181-92.

3. Umehara H, Okazaki K, Masaki Y, et al. Comprehensive diagnostic criteria for IgG4-related disease (IgG4-RD), 2011. Mod Rheumatol 2012; 22: 21-30.

4. Okazaki K, Umehara H. Are classification criteria for IgG4-RD The concept ofIgG4-Related Disease and proposal of comprehensive diagnostic criteria in Japan. Int J Rheumatol 2012; 2012: 357071.

5. Shimosegawa T, Chari ST, Frulloni L, et al. International consensus diagnostic criteria for autoimmune pancreatitis: guidelines of the international association of pancreatology.Pancreas 2011; 40: 352-8.

6. Kawano M, Saeki T, Nakashima H, et al. Proposal for diagnostic criteria forIgG4-related kidney disease. Clin Exp Nephrol 2011; 15: 615-26.

7. Moriyama M, Ohta M, Furukawa S, et al. The diagnostic utility of labial salivary gland biopsy in IgG4-related disease. Mod Rheumatol 2016; 3: 1-5.

8. Lang D, Zwerina J, Pieringer H. IgG4-related disease: current challenges and future prospects. Ther Clin Risk Manag 2016; 12 189-99.

9. Nishimoto N, Terao K, Mima T, et al. Mechanisms and pathologic significance in the increase in serum interleukin-6 (IL-6) and soluble IL-6receptor after administration of an anti-IL-6 receptor antibody, tocilizumab,in patients with rheumatoid arthritis and Castleman disease. Blood 2008;112: 3959-64.

10. Sato Y, Kojima M, Takata K, et al. Multicentric Castleman's disease with abundant IgG4-positive cells: a clinical and pathological analysis of six cases. J Clin Pathol 2010; 63: 1084-9.

11. Rose-John S, Scheller J, Elson G et al: Interleukin-6 biology is coordinated by membrane-bound and soluble receptors: role in inflammation and cancer. J Leukoc Biol 2006; 80: 227-36.

12. Masaki Y, Kurose N, Yamamoto M, et al. Cutoff values of serum IgG4 and histopathological IgG4+ plasma cells for diagnosis of patients with IgG4-related disease. Int J Rheumatol 2012; 2012: 580814.

13. Levi-Schaffer F, Weg VB. Mast cells, eosinophils and fibrosis. Clin ExpAllergy 1997; 27: 64-70.

14. Mizushima I, Yamada K, Fujii H, et al. Clinical and histological changes associated with corticosteroid therapy in IgG4-related tubulointerstitial nephritis. Mod Rheumatol 2012; 22: 859-70.

15. Nakajima A, Masaki Y, Nakamura T, et al. Decreased expression of innate immunity-related genes in peripheral blood mononuclear cells from patients with IgG4-related disease. PLoS One 2015; 10: e0126582.

16. Ahn JK, Huang B, Bae EK, et al. The role of $a$-defensin-1 and related signal transduction mechanisms in the production of IL-6, IL-8 and MMPs in rheumatoid fibroblast-like synoviocytes. Rheumatology 2013; 52: 1368-76.

17. Hirano T, Akira S, Taga $\mathrm{T}$, et al. Biological and clinical aspects of interleukin 6. Immunol Today 1990; 11: 443-9.

18. Kimura A, Kishimoto T. IL-6: regulator of Treg/Th17 balance. Eur J Immunol 2010; 40: 1830-5.

19. Zen Y, Fujii T, Harada K, et al. Th2 and regulatory immune reactions are increased in immunoglobin G4-related sclerosing pancreatitis and cholangitis. Hepatology 2007; 45: 1538-46. 